\title{
Evaluation of the Capability of the Modified Transtibial Technique in Restoring a Near Anatomic Anterior Cruciate Ligament Reconstruction
}

\author{
AHMED N. MORRA, M.D.; HESHAM MESBAH, M.D.; HAZEM FAROUK, M.D.; \\ EHAB A. HUSSEIN, M.D. and HOSSAM EL-DIN E. MOHAMMED, M.D.
}

The Department of Orthopaedic Surgery, Faculty of Medicine, Cairo University

\begin{abstract}
Background: ACL reconstruction becomes a very common procedure today. There are many techniques to put the femoral and tibial tunnels in a near anatomic position that allow regaining knee function and prevent malpositioning of the tunnels which may results in graft failure. Our hypothesis suggests that modified transtibial technique (MTT) will give comparable measures to native ACL.
\end{abstract}

Purpose: The aim of this paper is to study the effect of modifications on the transtibial technique regarding the graft positions \& angles and their impact on clinical outcome.

Patients and Methods: The study based on 30 patients, started from February 2013 to December 2014. All patients are males aged between 19-39 years (mean is 28.4 years with $5.0 \pm \mathrm{SD}$ ) having torn ACL underwent arthroscopically-assisted ACL reconstruction using hamstring tendons and a modified transtibial technique.

Results: Our "MTT" for single bundle ACL reconstruction showed comparable results to traditional TT. Ideally, our MTT graft was coronally inclined similar to that of the native ACL. It was also found to be more coronally oblique than the graft in traditional TT. These radiological results should be correlated with the clinical outcomes in order to note whether or not these improved radiological measurements would result in improved functional and clinical outcomes of the surgery.

Conclusion: This technique succeeded to put the graft in a more oblique position that presumably leads to improved clinical and functional outcome.

Key Words: Anterior Cruciate Ligament - MTT - Radiology - Arthroscopy - Orthopaedics.

\section{Introduction}

ARTHROSCOPIC single-bundle (SB) is the "gold standard" technique for anterior cruciate ligament reconstruction (ACLR) [1] . It is a common proce-

Correspondence to: Dr. Ahmed N. Morra,

The Department of Orthopaedic Surgery, Faculty of Medicine, Cairo University dure that has success rates of 83 to $95 \%$. However, some still debates the superiority of double-bundle ACL reconstruction due to its proposed biomechanical advantages [2]. Recently, it has been postulated that the SB transtibial ACL reconstruction places the graft in a non-anatomical femoral insertion site [3]. Given that the most common cause of ACL reconstruction failure has been the non- anatomical femoral tunnel placement, [4] the anteromedial portal technique (AM) emerged as a potential solution for non-anatomical reconstructions. It has been suggested as a method to place the graft in an anatomical position and thus improve rotational stability, without increased complexity $[3,5]$. The use of transtibial techniques (TT), has thus been largely abandoned as the position of the femoral tunnel is dictated by the tibial tunnel, without a greater freedom for the surgeon to place the graft in the anatomical position $[5,6]$. Several authors have tried afterwards to remodify the ordinary transtibial techniques in order to position the femoral tunnel more anatomically. Modifications are primarily directed to positioning the femoral tunnel more inferiorly increasing the graft inclination aiming at better radiological tunnel position and subsequent better clinical stability of the knee joint after surgery.

We hypothesize that a modified TT can result in better tunnel position that restores the knee stability after ACL reconstruction without using AM portal that makes it easier for surgeons commonly using TT techniques.

\section{Patients and Methods}

From February 2013 to December 2014, thirty patients underwent ACL reconstruction in the Orthopaedic Department of Kasr Al-Aini Hospital, 
Cairo University. A prospective study was undertaken to assess the results of arthroscopicallyassisted ACL reconstruction using hamstring tendons and a modified TT technique. Our study population were males aged between 19-39 years (mean is 28.4 years with $5.0 \pm \mathrm{SD}$ ). All young active patients with ACL tear and consequent functional instability were included in the current study. Patients with osteoarthritis of the ipsilateral knee, or aged less than 18 years, or with other knee ligamentous injuries were excluded from this study. Time interval between injury and operation ranged from 1-23 months. The mean interval was 8.3 months with $5.0 \pm \mathrm{SD}$.

Eight patients out of the 30 have had a meniscal injury confirmed during the arthroscopy. Six patients had their surgeries done after the mean interval of 8.5 months. Partial Meniscectomy was done in 7 while one case has had subtotal meniscectomy. The patients have been evaluated either subjectively, objectively, instrumentally, functionally, and radiologically.

The subjective assessment form was done using the IKDC subjective assessment form [7] and the Tenger-Lysholm Knee Scoring system [8]. These two systems were expressed as a percentile score. The patients were scored pre operatively at the same day of surgery then postoperatively with every postoperative visit. The results of these scoring systems at the end of one year were considered the final results. Radiological assessment consists of certain measurements are assessed on the MRI, X-ray AP view and lateral view. These radiological measurement include:

- Sagittal intact ACL angle: $[\mathbf{9 , 1 0}]$

This angle is measured on the sagittal cut of the MRI of the normal knee (Fig. 1).

\section{- Graft inclination angle: $[\mathbf{9 , 1 0 ]}$}

This angle is measured from the anteroposterior view in X-rays (Fig. 2).

\section{- Femoral graft angle (FGA) : [9]}

In the A-P view of X-ray knee, the angle between the axis of femoral tunnel andthe joint line is defined as the FGA (Fig. 3).

\section{- Tibial graft angle (TGA): [9]}

In the lateral view of X-ray knee, the angle between the axis of the tibial tunnel and a line perpendicular to the long axis of the tibia is defined as the (FGA), (Fig. 4). This angle is compared to the sagittal ACL angle.

\section{Surgical treatment:}

Preoperative assessment by history taking, general and local examination using the subjective scores and the IKDC evaluation forms were done. They were admitted on the same day of surgery and discharged later that day.

On the operative table, patients lie supine with their operated upon knees were flexed at the side edge of table. Examination under anesthesia was done to confirm preoperative diagnosis then a tourniquet was applied to the upper thigh. Then, the knee and leg were sterilized and draped.

\section{Technical steps:}

\section{1- Diagnostic arthroscopy:}

Routine diagnostic arthroscopy is performed initially to confirm the injury and evaluate other pathological conditions. The ACL stump was debrided as required for adequate visualization. Overthe-top position must be clearly visualized. ACL fibers that were not obstructive are left intact for possible vascular and cellular ingrowth.

\section{2- Tendons harvesting \& preparation:}

The hamstrings (semitendenosis and gracilis) tendons were harvested through a $3-4 \mathrm{~cm}$ vertical incision avoiding injury to the infrapatellar nerve. This incision was also used for drilling of the tibial bone tunnel. The two tendons were then cleaned, debrided and looped together around a free suture in a quadruple way and sutured using a whip stitch suture. The graft diameter and length were then measured and mounted on the traction device and covered with a wet gauze.

\section{3- Tibial tunnel drilling: [11,12]}

This step was of crucial importance in such a technique. It was a triangle formed by three landmarks; the posterior extension of the anterior horn of lateral meniscus, the medial tibial eminence, and the anterior border of the posterior cruciate ligament.

Same landmarks were used, only with few modifications as follows:

a- Tibial tunnel starting point at the proximal tibia started at about $15-20 \mathrm{~mm}$ below the medial plateau and about $15 \mathrm{~mm}$ from medial edge of tibial tubercle.

b- Tibial drill guide 40 degrees.

c- Tibial drill guide 40 degrees to the tibial long axis in coronal plane.

d- Intraarticular guide tip anterior and medial to the conventional site. 
e- Remove bone from the posterolateral corner of tibia tunnel, posterolateral corner widening.

\section{4- Femoral tunnel drilling: [11,12]}

After drilling the tibial tunnel, a femoral guiding device was introduced from the tibial tunnel. Some modifications allowed more anatomic inclination to the femoral tunnel which are:

a- Posterolateral notchoplasty contouring the roof and superolateral corner of the femoral notch to resemble a Roman Arch. This will clean any osteophytes and bonny edges and allow more free positioning of the femoral drill guide.

b- Femoral guiding device should be $2 \mathrm{~mm}$ smaller than the size of tibial tunnel to allow more free mobility in the tunnel. This allowed more inferior positioning of the guide and more oblique inclination of the graft.

c- External rotation of the femoral guide after hooking its shoulder over the top position will give extra few degrees of obliquity (Fig. 5).

\section{5- Graft Advancement \& Fixation:}

Graft advanced through femoral tunnel and suspended using endbutton, while graft fixation in the tibial side is done after successive cycling of the knee under maximal graft tension then a biodegradable interference screw 1-2mm larger than the size of the graft was inserted with the knee in 20 degrees flexion and the tibia was pressed firmly backwards.

\section{6- Wound irrigation, closure and dressing:}

After Surgery, patients were discharged after a minimum of 6 hours at the same day. Before discharge, physiotherapists gave them the postoperative instructions regarding mobilizations, and their rehabilitation schedule. They took home medications, physiotherapy appointment, and first postoperative clinic appointment after two weeks just to check wounds and to remove sutures.

All patients were subjected to the same rehabilitation program that started on the same day of surgery with some anti-inflammatory measures and weight bearing as tolerated was encouraged. In the early phase that lasts 6 weeks after surgery, training for knee flexors tensioning along with knee extensor isometric closed chain exercises 050 on the first 2 weeks followed by open chain $50-100$ after wards. Intermediate phase (6- $12 \mathrm{w})$ started with the focus went to resolve any impairment like resolving sweeping and improving resting ROM with emphasis on extension. Also closed kinetic chain exercises such as leg presses and squats were instructed. Neuromuscular training and balancing exercises were highly emphasized in this phase till 12 weeks after surgery were completed. In the late phase that follows the focus went to regaining dynamic joint stability to allow functional movement pattern and allow a safe return to sports by the end of this phase.

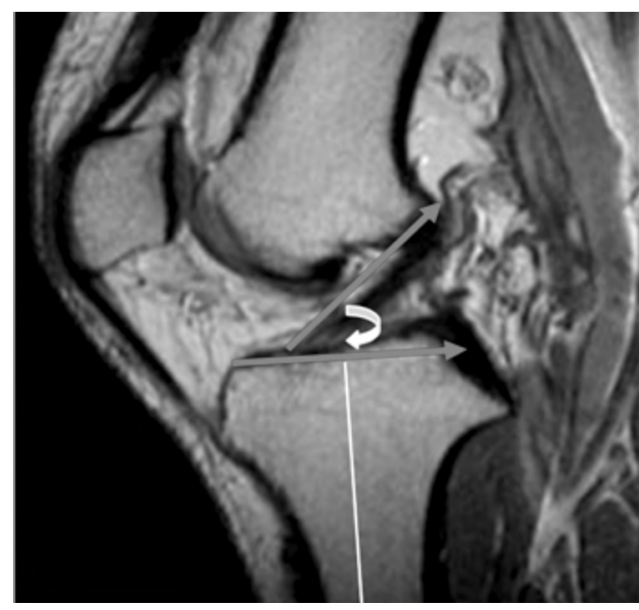

Fig. (1): Sagittal intact acl angle $[9,10]$.

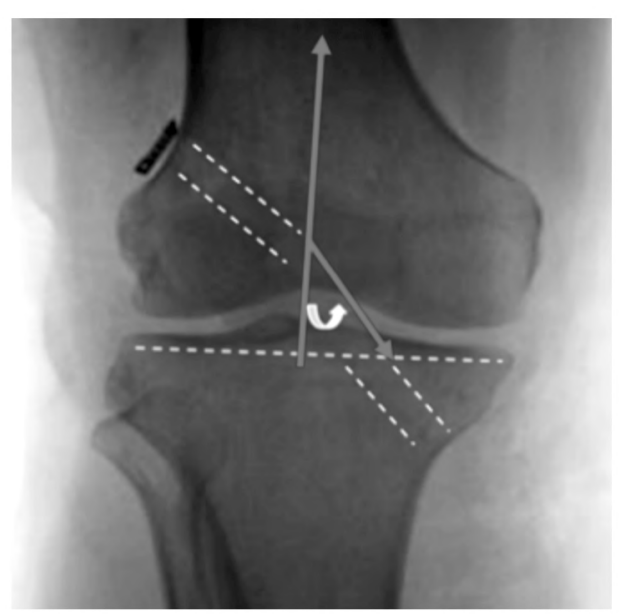

Fig. (2): Graft inclination angle $[\mathbf{9 , 1 0}]$

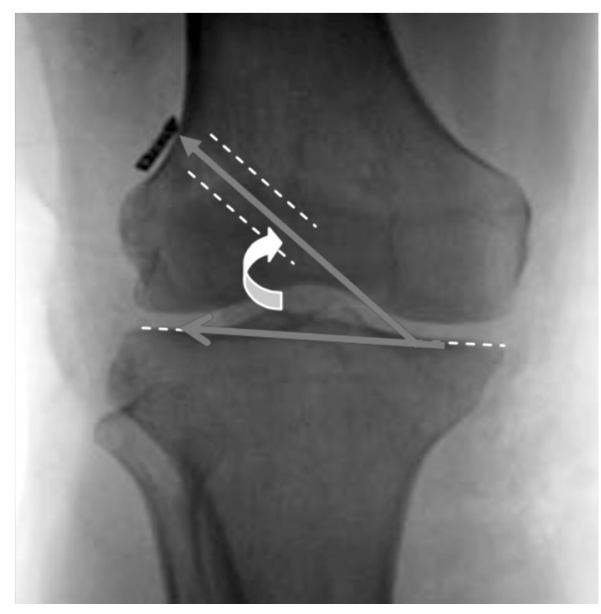

Fig. (3): Femoral graft angle [9] 


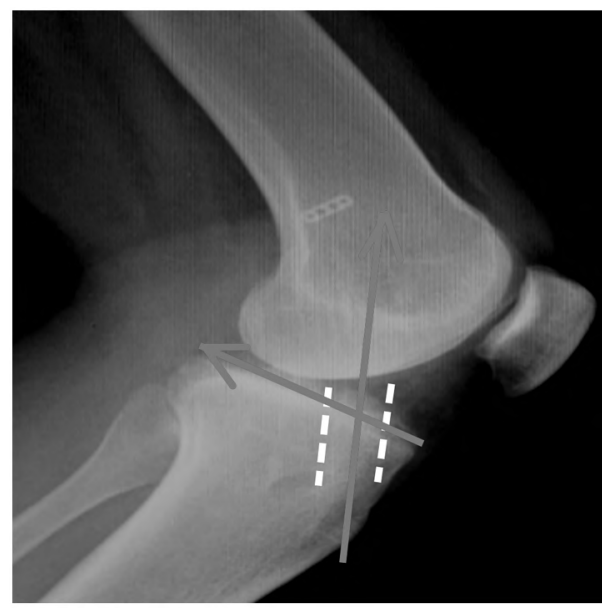

Fig. (4): Tibial graft angle [9].
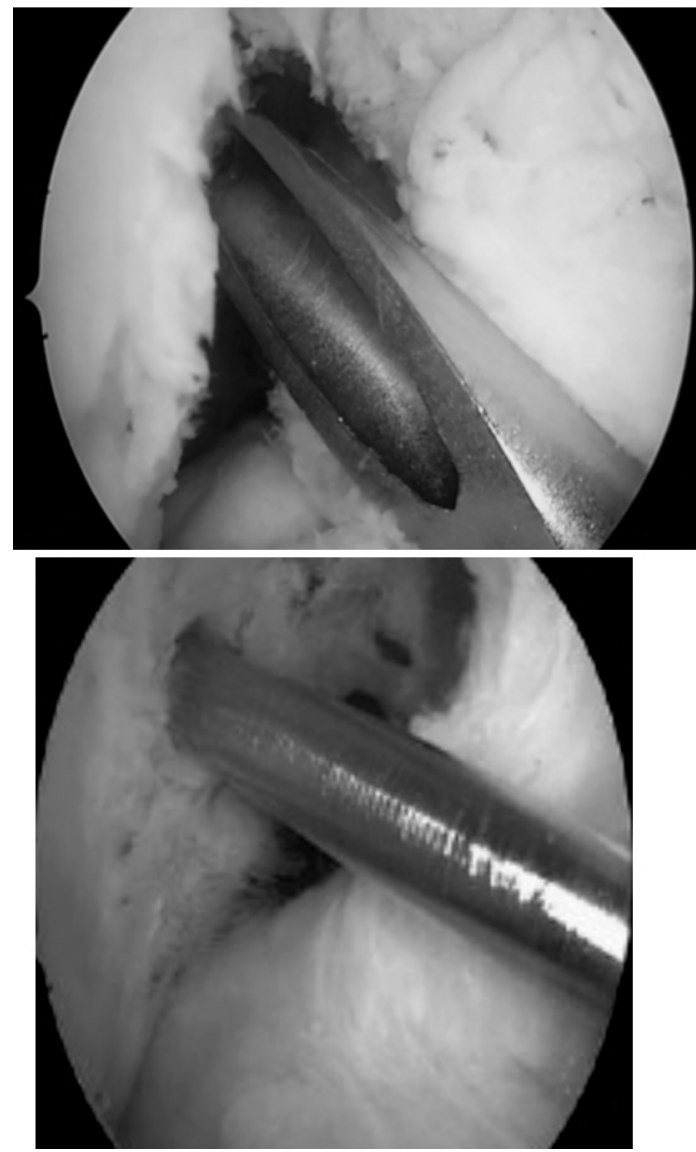

Fig. (5): External rotation of femoral guide.

\section{Results}

Data were analyzed using Statistical Program for Social Science (SPSS) version 18.0. Quantitative data were expressed as mean \pm standard deviation (SD). Qualitative data were expressed as frequency and percentage.

- Ikdc score \%:

Subjective IKDC scores for all patients showed improvements comparing pre to postoperative scores. Preoperative mean score was $55 \pm$ SD 16.1 while postoperative mean score was 77.5 SD 7.4. Also Improvement was still evident at the final score at the end of the $12^{\text {th }}$ month $92.5 \pm$ SD 4.9 (Table 1).

Table (1): IKDC scores.

\begin{tabular}{lccc}
\hline & \multicolumn{3}{c}{ Modified TTT } \\
\cline { 2 - 4 } IKDC score & Mean \pm SD & $\begin{array}{c}\text { Unpaired } \\
t \text {-test }\end{array}$ & $\begin{array}{c}\text { One way } \\
\text { ANOVA test }\end{array}$ \\
\hline Preoperative & $55.0 \pm 16.1$ & & \\
Mean postoperative & $77.5 \pm 7.4$ & $p<0.0001$ & $p<0.05$ \\
Final postoperative & $92.5 \pm 4.9$ & & \\
\hline
\end{tabular}

Lysholm scores as well, showed significant improvement from a preoperative mean score of 55.4 (SD 14.5) to a postoperative mean score of 81.1 (SD 6.4) and a final score of 93. 1 (SD 7.0).

Final evaluations denoting significant improvement in these aspects for the knee joint comparing the pre to postoperative evaluation. Other aspects of objective evaluation regarding compartment or $\mathrm{X}$-ray findings showed that more than $90 \%$ of the cases were graded either normal or nearly normal according to the IKDC objective grading system and the Chi-square test had a $p$-value of $>0.5$ which revealed clearly an insignificant difference between pre and postoperative evaluations.

Functionally, the one leg hop test showed 28 patients $(93 \%)$ had their knees, after 12 months postoperative, graded again as normal or nearly normal and only 2 patients could not successfully achieve more than $75 \%$ of the distance covered by the normal side. This looked significant knowing that $50 \%$ of cases were graded abnormal in the preoperative evaluation. The Chi-square test had a $p$-value of 0.000194 which denoted an extremely significant difference.

\section{- Relation between ligament examination and} LYSHOLM \& IDKC final scoring system:

The final score of both Lysholm and IKDC subjective scoring systems showed a highly significant relationship to the ligamentous examination of the knee, $p$-value $<0.001$.

\section{- Correlation between interval before surgery and LYSHOLM \& IKDC final scoring system:}

Among the patients, there was a highly significant relation between the del ay in surgical intervention "months" and the subjective knee scores "LYSHOLM \& IKDC" prior to surgery, $p$-value $<0.001$. The longer the time since injury, the more worse was the preoperative presentation. 


\section{- Complications:}

Postoperative complications were reported in four cases (3.3\%). Intraoperatively, a case of graft impingement was encountered where a minimal notchoplasty was performed to widen the roof top of the notch abolishing such impingement.

\section{Discussion}

ACL is the most commonly injured ligament in the knee [13]. Surgical treatment is considered the predominant line of treatment for ACL tears and is recommended for all young active individuals especially athletes.

Several studies have confirmed that better knee joint stability, graft positioning and obliquity may result from anatomical positioning of the bone tunnels during single-bundle anterior cruciate ligament (ACL) reconstruction compared to the unfavourable isometric vertical positioning of the bone tunnels [14].

Other studies found no significant difference. In their systemic review, Chalmers et al., [15] stated that "The AM portal technique for ACL reconstruction may be more likely to produce improved clinical and biomechanical outcomes but that the TT technique is capable of producing similar outcomes". On the other hand, Riboh et al., [16] in their larger systematic review found that, while there are biomechanical data suggesting improved knee joint stability and more anatomic graft placement with AM technique, no significant clinical differences were found between AM and TT techniques. In the current study we offered a modification of the traditional TT in simplified steps to add more obliquity and near anatomic position of the graft femoral insertion, suggesting that this would result in better Knee joint stability.

The modified transtibial ACL reconstruction could improve the patient subjective assessment of the knees post operatively throughout the followup period until the final evaluation at the 12 months postoperative. The Preoperative mean IKDC score was 55\% improving significantly at the final assessment to $92.5 \%$. This is significantly better than the mean IKDC score postoperatively of numerous TT studies reporting a mean postoperative IKDC range $78-90 \%$ in three different studies by Lee et al., Hussein et al., and Mardani-Kivi et al., [17].

The subjective scoring of the patients in the preoperative state was found to affect significantly both the mean and the final scores for the patients. Patients with better pre-operative subjective state would have smoother postoperative period and better final outcome. This may raise the importance of good pre-operative rehabilitation and early surgical intervention.

Regarding the radiological angles, the coronal graft inclination angle "GIA" in the current study group with a modified TT technique, had a "mean of $23.76^{\circ} \pm 5.64$ SD", which puts the graft in more inclination than native ACL that was reported by Jonathan et al., [18] to be $15.72^{\circ} \pm 4.75$ SD. Our MTTT had an angle of femoral tunnel "FGA$50.2^{\circ} \pm 9.5 \mathrm{SD}$ " which is more oblique than anatomic ranges " $60.7^{\circ} \pm 4.2 \mathrm{SD}$ " [19] but less than other modified techniques " $42.5^{\circ} \pm 6.1$ SD" [20] . Significantly, it is still far oblique than the vertical angles found in non-anatomic ranges " $82.8^{\circ} \pm 12.5$ SD" and traditional TT techniques " $85.8^{\circ} \pm 9.3$ SD" [21]

The MTTT in the current study succeeded to put the graft and tunnel in more obliquity than the traditional non anatomic TTT and even more than anatomic ranges. The patient complaints and subjective scoring were found to be strongly related to the graft stability "ligamentous examination" $p$-value $<0.001$. This means that, the more stable were the grafts as indicated by higher grades of ligamentous examination in the IKDC examination form, the less were the complaints detected in the subjective forms "LYSHOLM \& IKDC".

The ability of the patients to achieve higher scores in the functional one leg hop test was affected by their subjective scores and subsequently by their graft stability " $p$-value $<0.05$ ".

\section{Conclusion:}

Our study offers a modified technique for those who prefer or being more accustomed on the transtibial technique. These simple modifications allow a near anatomic TTT with better results compared to the traditional TTT in many aspects or at least similar to it in the remaining with high safety and few complications.

\section{References}

1- DUQUIN T.R., WIND W.M., FINEBERG M.S., SMOLINSKI R.J. and BUYEA C.M.: Current trends in anterior cruciate ligament reconstruction. J. Knee. Surg., 22:7-12. doi: 10.1055/s-0030-1247719, 2009.

2- BUSS M.D., WARREN R.F., WICKIEWICZ T.L., GALINAT B.J. and PANARIELLO R.: Arthroscopically assisted reconstruction of the anterior cruciate ligament with use of autogenous patellar-ligament grafts. results after twenty-four to forty-two months. J. Bone. Joint. Surg. Am., 75: 1346-1355, 1993.

3- ARNOLD M.P., KOOLOOS J. and KAMPEN A.: Singleincision technique misses the anatomical femoral anterior 
cruciate ligament insertion: A cadaver study Knee. Surg. Sports. Traumatol. Arthrosc., 9: 194-199, 2001.

4- GEORGOULIS ANASTASIOS D., RISTANIS STAVROS, CHOULIARAS VASILIS, MORAITI CONSTANTINA O. and STERGIOU NICHOLAS: " Anterior cruciate ligament reconstruction with a quadrupled hamstring autograft does not restore tibial rotation" Journal Articles. Paper, 107, 2005.

5- BOTTONI C.R., ROONEY R.C., HARPSTRITE J.K. and KAN D.M.: Ensuring accurate femoral guide pin placement in anterior cruciate ligament reconstruction. Am. J. Orthop., 27: 764-766, 1998.

6- CHA P.S., CHHABRA A. and HARNER C.D.: Singlebundle anterior cruciate ligament reconstruction using the medial portal technique. Oper. Tech. Orthop., 15: 8995, 2005.

7- http://www.orthopaedicscore.com . $2000 \mathrm{ikdc}$ knee form.

8- http://www.orthopaedicscore.com . tegner lysholm knee scoring scale.

9- MICHAEL ELIAS, et al.: Differences in graft orientation using the transtibial and anteromedial portal technique in anterior cruciate ligament reconstruction: A magnetic resonance imaging study. Knee. Surg. Sports. Traumatol. Arthrosc., 17: 880-886, 2009.

10- NIKOLAOS GOUGOULIAS, et al.: Acl reconstruction: can the transtibial technique achieve optimal tunnel positioning? a radiographic study. The Knee, 15: 486490, 2008

11- DANA P. PIASECKI M.D. and BERNARD R. BACH M.D.: Anatomical single bundle anterior cruciate ligament reconstruction with a transtibial technique. The American Journal of Orthopedics, June, 302-304, 2010.

12- JOHNSON D.L., SWENSON T.M., IRRGANG J.J. and HARNER C.D.: Revision anterior cruciate ligament surgery: Experience from pittsburgh. Clin. Orthop. Relat. Res., 325: 100-109, 1996.

13- RAINER SIEBOLDI, DAVID DEJOUR and STEFANO ZAFFAGNIN.: Acl reconstruction, A Practical Surgical Guide. Springer. Esska., 2014.
14- JONG-KEUN, et al.: Can anatomical femoral tunnel position improve clinical outcomes in anterior cruciate ligament reconstruction. sports medicine and arthroscopy. Paper. Feb., 102: 1034, 2010.

15- CHALMERS P.N., MALL N.A., COLE B.J., et al.: Anteromedial versus transtibial tunnel drilling in anterior cruciate ligament reconstructions: A systematic review. Arthroscopy, 29: 1235-1242, 2013.

16- RIBOH J.C., HASSELBLAD V., GODIN J.A., et al.: Transtibial versus independent drilling techniques for anterior cruciate ligament reconstruction: A systematic review, meta-analysis, and metaregression. Am. J. Sports Med., 41: 2693-2702, 2013.

17-HUSSEIN M., VAN ECK C.F., CRETNIK A., DINEVSKI D. and FU FH: Prospective randomized clinical evaluation of conventional single-bundle, anatomic single-bundle, and anatomic double-bundle anterior cruciate ligament reconstruction: 281 cases with 3- to 5-year follow-up. Am. J. Sports Med., 40: 512-520, 2012.

18- JONATHAN REID B.A.: Detrmining the angle of inclination of the native acl in the coronal and sagittal plane tria orthopedic center (mn), www.ahc.umn.edu ., 2015.

19- ILLINGWORTH K.D., HENSLER D., et al.: Acl inclination angle on mri and femoral tunnel angle on ap xray: a comparison study with tunnel positions on $3 \mathrm{~d}$ ct in single bundle acl reconstruction. Ors Annual Meeting, 2011.

20- YOUM Y.S. ${ }^{1}$, CHO S.D. ${ }^{2}$, LEE S. H. ${ }^{1}$ and YOUN C.H. ${ }^{1}$ Modified transtibial versus anteromedial portal technique in anatomic single-bundle anterior cruciate ligament reconstruction: Comparison of femoral tunnel position and clinical results. Am. J. Sports. Med. Dec., 42 (12): 2941-7. doi: 10. 1177/0363546514551922. epub 2014 sep 30, 2014.

21- D. VERMESAN and F. INCHINGOLO.: Anterior cruciate ligament reconstruction and determination of tunnel size and graft obliquity. European review for Medical and Pharmacological Sciences, 19: 357-364, 2015. 


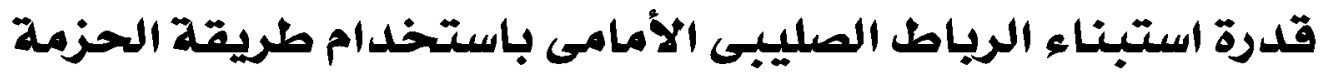

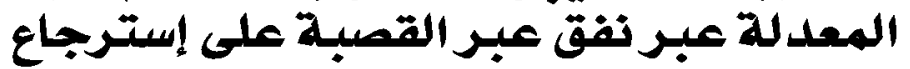 الوضع المثالى للرباط العبد}

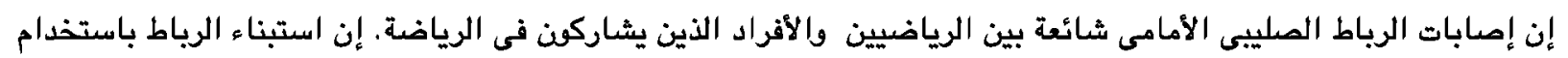

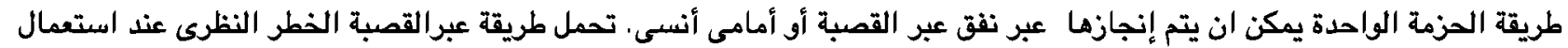

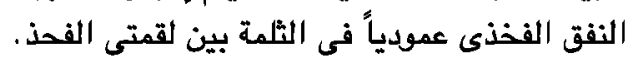

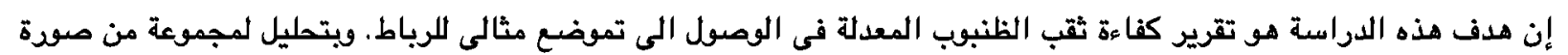

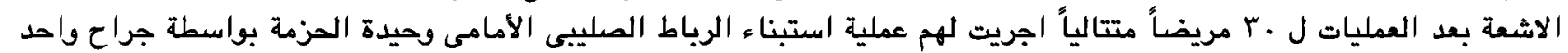

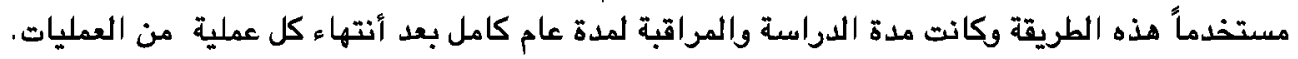

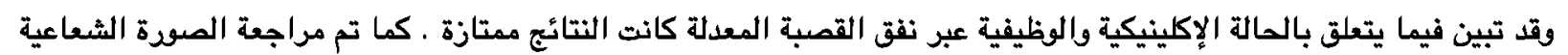

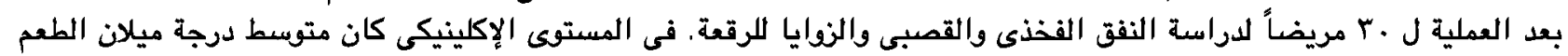

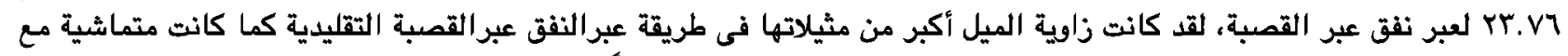

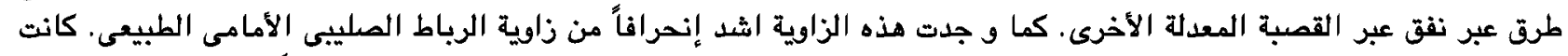

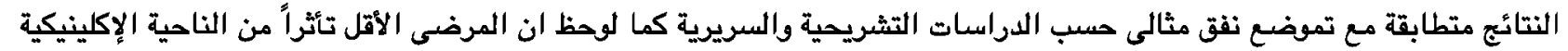

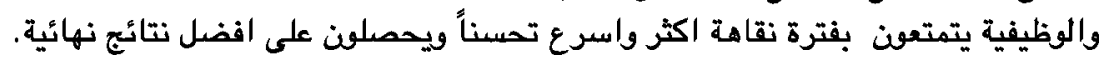

